\title{
Cardiac Function in Long-Term Survivors of Childhood Lymphoma
}

\author{
Mark K. Friedberg, ${ }^{1}$ Ido Solt, ${ }^{2}$ Myriam Weyl-Ben-Arush, ${ }^{3}$ Yulia Braver, ${ }^{4}$ \\ and Avraham Lorber ${ }^{4}$
${ }^{1}$ Department of Pediatrics, Meyer Children's Hospital, Rambam Medical Center, Technion-Israel Institute of Technology, Haifa 31096, Israel
${ }^{2}$ Division of Obstetrics and Gynecology, Meyer Children's Hospital, Rambam Medical Center, Technion-Israel Institute of Technology, Haifa 31096, Israel
${ }^{3}$ Department of Pediatric Hematology and Oncology, Meyer Children's Hospital, Rambam Medical Center, Technion-Israel Institute of Technology, Haifa 31096, Israel
${ }^{4}$ Unit of Pediatric Cardiology, Meyer Children's Hospital, Rambam Medical Center, Technion-Israel Institute of Technology, Haifa, 31096, Israel

Correspondence should be addressed to Avraham Lorber, A_lorber@rambam.health.gov.il

Received 3 November 2010; Revised 4 January 2011; Accepted 4 January 2011

Academic Editor: Dirk Westermann

Copyright ( $) 2011$ Mark K. Friedberg et al. This is an open access article distributed under the Creative Commons Attribution License, which permits unrestricted use, distribution, and reproduction in any medium, provided the original work is properly cited.

Objectives. We studied long-term effects of therapy for childhood lymphoma on cardiac function. Design and patients. We prospectively evaluated 45 survivors of childhood lymphoma, using clinical parameters, electrocardiography and echocardiography. Further comparisons were made between lymphoma subgroups and between males and females. Results. Mean age at diagnosis was 9.1 years. Mean followup duration was 10.9 years. The NYHA functional class was I in 43 patients and II in 2 patients. A prolonged QTc interval $(>0.44 \mathrm{msec})$ was found in 8 patients. Left ventricular $(\mathrm{LV})$ systolic function and compliance were normal (LV shortening fraction $40 \pm 5.6 \%$; cardiac index $2.84 \pm 1.13 \mathrm{~L} / \mathrm{min} / \mathrm{m}^{2}$; E/A wave ratio $2.5 \pm 1.3$; mean \pm S.D.), $\mathrm{LV}$ mass was normal $\left(97 \pm 40\right.$ grams $/ \mathrm{m}^{2}$, mean \pm S.D.). Mitral regurgitation was observed in $7 / 45$ patients $(16 \%)$. Asymptomatic pericardial effusions were found in $3 / 45$ (7\%) patients. Conclusions. Long-term follow-up shows that most parameters of cardiac function are normal in survivors of childhood lymphoma. This is likely due to relatively low doses of anthracyclines in modern protocol modalities. Abnormalities in mitral valve flow, QTc prolongation and in a small proportion of survivors, and functional capacity necessitate long-term cardiac follow-up of these patients.

\section{Introduction}

Increased long-term survival following effective treatment of childhood lymphomas has placed greater emphasis on residual sequelae of therapy for lymphoma and the impact of these sequelae as the cause of further morbidity and mortality. Following treatment of childhood Hodgkin's disease, cardiac pathology is second only to neoplasm as the cause of death in initial survivors [1]. As the number of cancer survivors increases, so does the number of patients exposed to therapies toxic to the heart and lung, including radiation and chemotherapeutic modalities that employ anthracyclines [2]. The literature is replete regarding effects of anthracyclines and radiation on the heart; however, there is still debate regarding the detection of cardiac sequelae [3], their significance [4], and the relation between different treatment modalities and the development of cardiac sequelae. We hypothesized that protocols employing anthracyclines and radiation would adversely affect long-term cardiac function and that these effects would differ between survivors who received different treatment modalities. We therefore prospectively evaluated long-term cardiac function in 45 survivors of childhood lymphomas, using clinical parameters, electrocardiography, 
TABLE 1: Demographic characteristics of survivors by lymphoma subtype.

\begin{tabular}{lcccc}
\hline & HD & BL & NHNB & Total/Average \\
\hline Males $(n)$ & 14 & 7 & 5 & 26 \\
Females $(n)$ & 12 & 5 & 2 & 19 \\
Mean age at diagnosis (years) & 10.8 & 7.4 & 5.5 & 9.1 (range: $2.1-16.4$ ) \\
Treatment duration (years) & 0.9 & 0.8 & 1.7 & 0.94 \\
Age at follow-up (years) & 23.1 & 19.1 & 16.7 & 21 (range: $2.1-16.4$ ) \\
Followup duration (years) & 11.3 & 10.9 & 9.5 & 10.9 (range: $2.1-16.4)$ \\
\hline
\end{tabular}

and echocardiography and compared these parameters between lymphoma subtypes.

\section{Patients and Methods}

The study included survivors of histology-diagnosed childhood lymphomas who had at least 5 years of followup from termination of therapy, and who were less than 18 years of age at treatment initiation. These criteria identified 45 survivors of 108 children treated for lymphoma at the Pediatric Hematology and Oncology department of a tertiary care center during a nineteen-year period. Fifteen additional survivors meeting these inclusion criteria declined complete participation in the protocol. All participants underwent evaluation which included an interview to assess functional class (New York Heart Association), physical examination, 12-lead electrocardiography (ECG), and echocardiography. Data were taken from a single assessment at the last available follow-up.

Informed consent was obtained from all participants or their legal guardians.

2.1. Statistics. Statistical analysis was performed using a commercially available package (Sigmastat 5.0 Jandel Scientific, San Rafael, California). Comparisons between the different lymphoma subgroups were made using one way ANOVA or the Tukey correction of this test where normality failed, and where relevant by the Student's T-test or the Mann-Whitney rank sum test where normality failed. Statistical significance was set at $P<.05$.

2.2. Study Population. Characteristics of the study population including treatment duration and mean follow-up duration are presented in Table 1.

2.3. Treatment Modalities. Tumor characteristics and therapeutic modalities are detailed in Table 2 .

The BL subgroup was divided between those treated in earlier years with protocols (COMP) that did not include anthracyclines (7/12 patients), and those that were treated more recently (5/12 patients) with protocols (LMB, NCI) containing anthracyclines. These two subgroups did not differ significantly with regard to age at diagnoses $(6.7 \pm 3.7$; $8.30 \pm 5.1$ years, resp., $P=.52)$ or age at follow-up (21.2 \pm 4.2 ; $16.1 \pm 5.8$ years, $P=.1$ ).

The mean dose of doxorubicin (adriamycin), for the group as a whole was $25 \mathrm{mg} / \mathrm{m}^{2}$ per treatment, leading to a cumulative dose of $150 \mathrm{mg} / \mathrm{m}^{2}$ or a total cumulative dose of 225-250 mg.

All participants underwent evaluation which included an interview to assess functional class (New York Heart Association (NYHA)), physical examination, 12-lead ECG, and echocardiography. Cardiovascular physical examination, ECG, and echocardiography were performed by 2 senior staff pediatric cardiologists (A. Lorber and Y. Braver). Analyses of ECG and echocardiography images were then undertaken in a blinded manner by different investigators (M. Friedberg and I. Solt). Stroke volume was calculated by multiplying the aortic valve area, measured from the 2dimensional echocardiographic long axis view of the internal aortic diameter at valvular level, with the velocity time integral (VTI) of aortic flow. Cardiac index (CI) was then calculated by multiplying this value by heart rate, and dividing the product by body surface area. Left ventricular indices were measured by M-mode echocardiography. Left ventricular mass was calculated by the Devereux method [5]. Left ventricular diastolic function was assessed from mitral valve inflow Doppler. Mitral regurgitation was graded according to the routine clinical gradation used in the lab as none, trivial, mild, moderate or severe. Echocardiographic data were compared to well-established normal values [6]. Normal values were defined as being within 2 standard deviations of the mean normal value: for $\mathrm{LV}$ ejection fraction $66 \pm 4 \%$, and LV fractional shortening $36 \pm 4 \%$, $\mathrm{LV}$ mass index $70.4 \mathrm{gram} / \mathrm{m}^{2}$ for males (10-95 percentile $48.5-103 \mathrm{gram} / \mathrm{m}^{2}$ ) and $60.7 \mathrm{gram} / \mathrm{m}^{2}$ for females (10-95 percentile $\left.35.6-81 \mathrm{gram} / \mathrm{m}^{2}\right)$. Early diastolic mitral inflow velocity ( $\mathrm{E}$ wave) to late diastolic mitral inflow velocity (A wave) ratio is $1.7 \pm 0.4$ to $2.5 \pm 0.9$. Cardiac dimensions were defined as being within 2 standard deviations of the normal mean for body surface area. A QTc $>0.44$ seconds was defined as prolonged.

\section{Results}

3.1. Functional Capacity and History. All patients had normal cardiovascular function prior to initiation of therapy.

The NYHA functional class was class I in 43 patients (96\%) and class II in 2 patients (4\%). These 2 patients did not have other comorbidities or notable toxic effects on followup. No Raynaud's phenomenon was reported. No events of near-syncope, syncope, or sudden death were noted. Clinical features of the cohort as a whole and of the Hodgkin's disease subgroup are shown in Table 3. 
TABLE 2: Lymphoma characteristics and treatment data of survivors by lymphoma subtype.

\begin{tabular}{|c|c|c|c|c|c|c|}
\hline Patient & Staging & Histology & ChemoTx & Irradiation & Total dose (cGy) & Mantle dose (cGy) \\
\hline \multicolumn{7}{|l|}{$\overline{H L}$} \\
\hline 1 & $3 B$ & NONE & MOPP & MANTLE & 1500 & 1500 \\
\hline 2 & $2 \mathrm{~B}$ & NS & MOPP & MANTLE & 3000 & 3000 \\
\hline 3 & $2 \mathrm{~B}$ & NS & MOPP & MANTLE & 4000 & 4000 \\
\hline 4 & $1 \mathrm{~A}$ & LP & MOPP & MANTLE & 2000 & 2000 \\
\hline 5 & $2 \mathrm{~B}$ & LP & MOPP & MANTLE & 4000 & 4000 \\
\hline 6 & $3 \mathrm{~A}$ & $\mathrm{MC}$ & MOPP/ABVD & TOT. NODAL & 1650 & 1500 \\
\hline 7 & $1 \mathrm{~A}$ & NS & MOPP & MANTLE & 2600 & 2800 \\
\hline 8 & $2 \mathrm{~A}$ & $\mathrm{MC}$ & MOPP/ABVD & MANTLE & 2000 & 2000 \\
\hline 9 & $2 \mathrm{~B}$ & MC & MOPP/ABVD & MANTLE,INV.Y.SPLEEN & 5800 & 4000 \\
\hline 10 & $2 \mathrm{~A}$ & NS & MOPP/ABVD & MANTLE & 1800 & 1800 \\
\hline 11 & $3 B$ & NS & MOPP/ABVD & MANTLE,PARAAORTIC & 8000 & 4000 \\
\hline 12 & $2 \mathrm{~B}$ & NS & MOPP/ABVD & MANTLE,INV.Y & 4000 & 4000 \\
\hline 13 & $2 \mathrm{~B}$ & $\mathrm{MC}$ & MOPP/ABVD & MANTLE & 3060 & 3060 \\
\hline 14 & $3 \mathrm{~B}$ & NS & MOPP & MANTLE,INV.Y,SPLEEN & 5100 & 2500 \\
\hline 15 & $2 \mathrm{~A}$ & NONE & MOPP & MANTLE,INV.Y & 8400 & 4400 \\
\hline 16 & 2B-BULKY & $\mathrm{MC}$ & MOPP/ABVD & MANTLE & 2600 & 2600 \\
\hline 17 & $3 \mathrm{~A}$ & NS & MOPP/ABVD & MANTLE & 2600 & 2600 \\
\hline 18 & 2A-BULKY & NS & MOPP/ABVD & MANTLE & 4000 & 4000 \\
\hline 19 & $1 \mathrm{~A}$ & NS & MOPP/ABVD & MINIMANTLE & 3600 & 3600 \\
\hline 20 & $2 \mathrm{~A}$ & NS & MOPP/ABVD & MANTLE & 3000 & 3000 \\
\hline 21 & $3 \mathrm{~B}$ & LD & MOPP & MANTLE,INV.Y & 8000 & 4000 \\
\hline 22 & $2 \mathrm{~A}$ & NS & MOPP & MANTLE & 4000 & 4000 \\
\hline 23 & $1 \mathrm{~A}$ & LP & NONE & MANTLE & 4000 & 4000 \\
\hline 24 & $2 \mathrm{~A}$ & NS & MOPP/ABVD & MANTLE & 2600 & 2600 \\
\hline 25 & $2 \mathrm{~A}$ & NS & NONE & MANTLE,PARAO,SPLEEN & 8000 & 4000 \\
\hline 26 & 2B-BULKY & NS & MOPP/ABVD & MANTLE & 3000 & 3000 \\
\hline Average & & & & & 3935 & 3152 \\
\hline \multicolumn{7}{|l|}{$\overline{B L}$} \\
\hline 27 & NP & 6 & COMP & & 0 & 0 \\
\hline 28 & $\mathrm{ABD}$ & 6 & $\mathrm{NCI}$ & & 0 & 0 \\
\hline 29 & $\mathrm{ABD}$ & 6 & LMB & & 0 & 0 \\
\hline 30 & $\mathrm{ABD}$ & 6 & COMP & & 0 & 0 \\
\hline 31 & $\mathrm{ABD}$ & 6 & COMP & & 0 & 0 \\
\hline 32 & $\mathrm{ABD}$ & 6 & COMP & & 0 & 0 \\
\hline 33 & $\mathrm{ABD}$ & 6 & $\mathrm{COM}$ & & 0 & 0 \\
\hline 34 & $\mathrm{ABD}$ & 6 & COMP & WHOLE ABDOMEN & 1550 & 0 \\
\hline 35 & $\mathrm{ABD}$ & 6 & $\mathrm{NCI}$ & & 0 & 0 \\
\hline 36 & $\mathrm{ABD}$ & 6 & LMB & & 0 & 0 \\
\hline 37 & NP & 6 & ACOMP & & 0 & 0 \\
\hline 38 & $\mathrm{ABD}$ & 6 & NCI & & 4000 & 0 \\
\hline Average & & & & & 462 & 0 \\
\hline \multicolumn{7}{|l|}{ NHNB } \\
\hline 39 & 0 & 7 & LSAL2 & & 0 & 0 \\
\hline 40 & 0 & 7 & LSAL2/COM & BRAIN,TOT.LUNG & 3600 & 0 \\
\hline 41 & 0 & 7 & LSAL2 & BRAIN,INV.Y & 5600 & 0 \\
\hline 42 & 0 & 7 & $\mathrm{COM}$ & & 0 & 0 \\
\hline 43 & 0 & 7 & LSAL2 & MEDIASTINUM & 750 & 0 \\
\hline 44 & 0 & 7 & LSAL2 & MEDIASTINUM & 2000 & 0 \\
\hline 45 & 0 & 7 & COMP & BRAIN & 2400 & 0 \\
\hline Average & & & & & 2050 & 0 \\
\hline
\end{tabular}

MOPP: nitrogen mustard, oncovin, prednisone, and procarbazine; COM/P: cyclophosphamide,oncovin, and methotrexate/prednisone; ABVD: adriamycin, bleomycin, vinblastine, and deticen; NCI: national cancer institute; ACOMP: like comp with adriamycine; LSA2L2: norma wollner protocol; LMB: no abbreviation; NS: nodular sclerosis; LP: lymphocyte predominance; MC: mixed cellularity; LD: lymphocyte depletion. 
TABLE 3: Electrocardiographic and echocardiographic data of all survivors and the Hodgkins disease subtype.

\begin{tabular}{|c|c|c|c|}
\hline & All & Hodgkins disease & \\
\hline \multicolumn{4}{|l|}{ Electrocardiography } \\
\hline Heart rate $(\mathrm{bpm})($ mean $\pm \mathrm{SD})$ & $77 \pm 11$ & & \\
\hline PR interval (seconds) (mean $\pm S D)$ & $0.144 \pm 0.024$ & & \\
\hline QTc interval (seconds) $($ mean \pm SD) & $0.411 \pm 0.032$ & $0.417 \pm 0.034$ & N.S \\
\hline \multicolumn{4}{|l|}{ Echocardiography } \\
\hline LV Fractional shortening (\%) & $40 \pm 5.6$ & $39.5 \pm 5.6$ & N.S \\
\hline Cardiac Index $\left(\mathrm{L} / \mathrm{min} / \mathrm{m}^{2}\right)$ & $2.84 \pm 1.13$ & $3.4 \pm 1.03$ & N.S \\
\hline $\operatorname{LVED}(\mathrm{cm})$ & $4.43 \pm 0.057$ & & N.S \\
\hline Septal thickness $(\mathrm{cm})$ & $0.65 \pm 0.12$ & $0.65 \pm 0.13$ & N.S \\
\hline Posterior wall thickness $(\mathrm{cm})$ & $0.66 \pm 0.1$ & $0.67 \pm 0.1$ & N.S \\
\hline $\mathrm{LV}$ mass $\left(\mathrm{gm} / \mathrm{m}^{2}\right)$ & $97 \pm 40$ & $96 \pm 38$ & N.S \\
\hline E/A wave ratio & $2.5 \pm 1.3$ & $2.29 \pm 1.09$ & N.S \\
\hline Mitral regurgitation $(n, \%)$ & $7 / 45(16)$ & $4 / 26(15)$ & N.S \\
\hline
\end{tabular}

3.2. Electrocardiography. All patients demonstrated normal sinus rhythm with no bradycardias or tachycardias noted. The percentage of survivors with a prolonged QTc interval by lymphoma subgroup is shown in Figure 1(a). A prolonged QTc interval was not associated with sinoatrial or atrioventricular node dysfunction-conduction anomalies.

Incomplete right bundle branch block was recorded in 4 patients (9\%, all diagnoses); complete right bundle branch block was recorded in 2 patients. No evidence of ischemia or "strain" was noted on nonstress ECG.

3.3. Echocardiography. Cardiac systolic and diastolic function was normal or low-normal for the group as a whole without significant differences between the subgroups (Table 3).

LV mass and shortening fraction did not correlate with mantle irradiation dose, QTc interval, or length of follow-up. Mean LV mass was within normal limits $(97 \pm$ 40 grams $/ \mathrm{m}^{2}$, mean \pm S.D.). LV mass was significantly higher for males as compared to females (all subjects, 112 grams versus 76 grams, resp.; $P<.01$ ). There was no significant difference in shortening fraction between males and females. Left ventricular end diastolic internal dimensions did not increase following relatively smaller shortening fraction values. Cardiac index and mass by lymphoma subgroup are presented in Figures 1(b) and 1(c), respectively.

Mild mitral regurgitation was observed in 7 of the 45 patients $(16 \%)$. The percentage of survivors with mitral regurgitation by subgroup is shown in Figure 1(d). Mitral valve prolapse was recorded in $1 \mathrm{HD}$ survivor. Pulmonary valve insufficiency, more than the accepted physiological norm, was found in 1 survivor of NHNB.

Asymptomatic small pericardial effusions were found in 3 of the 45 patients (7\%). No larger effusions or pericardial thickening was noted in any of the patients. A mitral inflow pattern consistent with constrictive or restrictive physiology (elevated E wave velocity, elevated E/A ratio, and short deceleration time) was not noted in any of the patients.

Selected electrocardiographic and echocardiographic parameters for the subset of BL survivors treated with anthracyclines versus those not treated with anthracyclines are presented in Table 4.

\section{Discussion}

Cardiac abnormalities described after treatment for childhood lymphomas can involve almost any aspect of cardiac function and include pericarditis, pericardial effusions, pericardial fibrosis, pancarditis, myocardial fibrosis with functional impairment, valvular disease, conduction defects, and coronary artery disease [1]. Although sequelae may develop at variable time intervals following treatment, emphasis is now placed on the long-term sequelae of lymphoma therapy $[7,8]$, which are caused mainly by anthracyclines and radiation [9].

In our study population, most parameters of cardiac status were within normal limits. This is most likely due to the relatively low dose of anthracyclines used. Previous study has shown that an average cumulative dose of $450 \mathrm{mg} / \mathrm{m}^{2}$ of doxorubicin causes a $23 \%$ abnormality rate in echocardiographic findings during a 7-year follow-up period [10] and that sequelae are rare at doses under $300 \mathrm{mg} / \mathrm{m}^{2}[10,11]$. Our study population received an average of $150 \mathrm{mg} / \mathrm{m}^{2}$ of doxorubicin, leading to a total dose of 225-250 mg, which is less than the average toxic dose. This is achieved in modern protocols through regimens employing multidrug therapy. The average follow-up duration for our population was 10.9 years, which is longer than that of many other studies, with relatively few cardiac sequelae from anthracyclines. However, these patients still warrant long-term cardiac follow-up, as the risk for cardiomyopathy continues to increase over time $[12,13]$. This vigilance is further justified by previous findings that patients may develop cardiac complications from anthracyclines, even when lower doses are used [13].

Studies performed 2 to 3 decades ago identified factors that augment the cardiotoxicity of anthracyclines. These include radiation of the mediastinum [14, 15], previous cardiac abnormalities, including involvement of the heart by the tumor [16, 17], uncontrolled hypertension $[11,12]$, and exposure to other chemotherapy modalities such as 
TABLE 4: Comparison of selected electrocardiographic and echocardiographic characteristics between Burkitt lymphoma survivors who received or did not receive anthracyclines as part of their treatment regimen.

\begin{tabular}{lccc}
\hline & B.L. patients who received anthracyclines & B.L. patients who did not receive anthracyclines & \\
\hline LV shortening fraction (\%) & $41 \pm 5$ & $39 \pm 3$ & N.S \\
QTc & $0.416 \pm 0.03$ & $0.4 \pm 0.02$ & N.S \\
LV mass & $121 \pm 36$ & $85 \pm 37$ & N.S \\
\hline
\end{tabular}

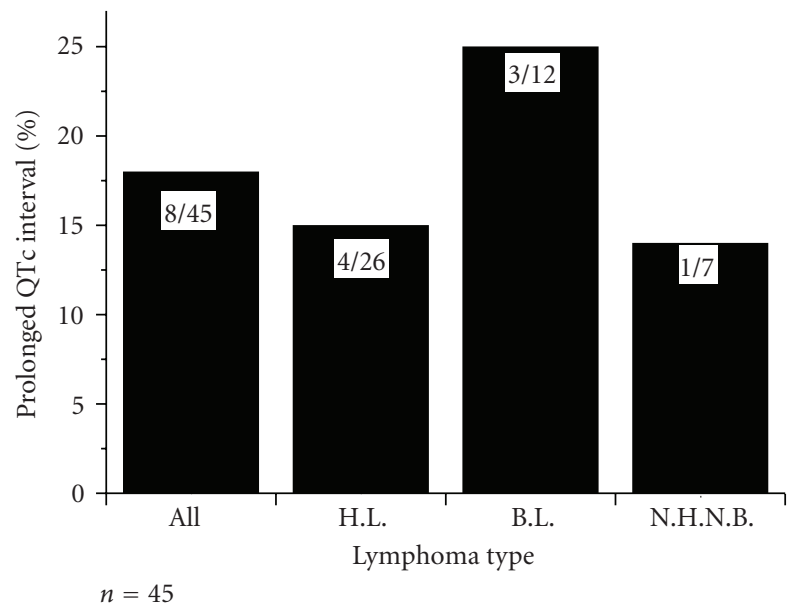

(a)

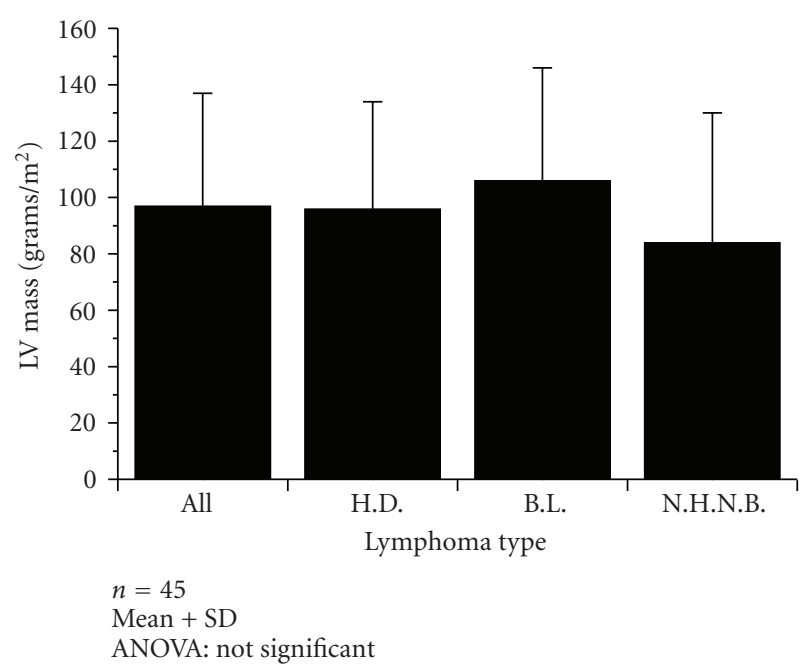

(c)

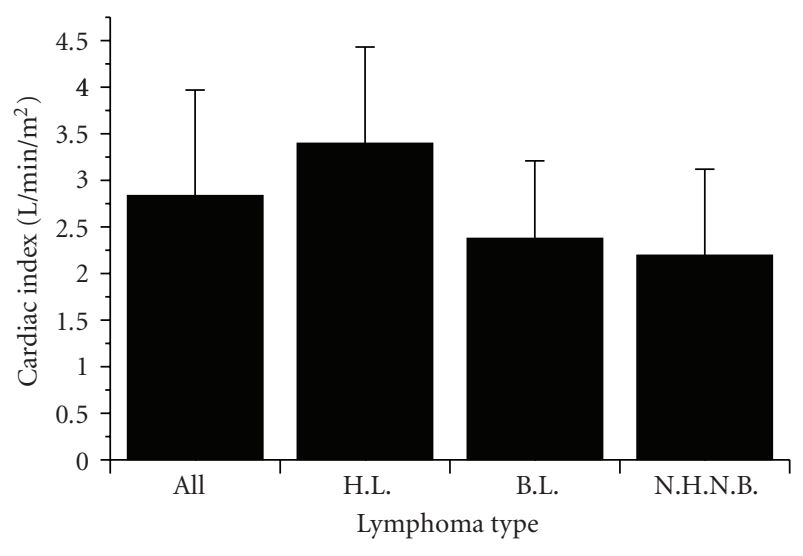

$n=45$

Mean + SD

ANOVA $P<.01$

(b)

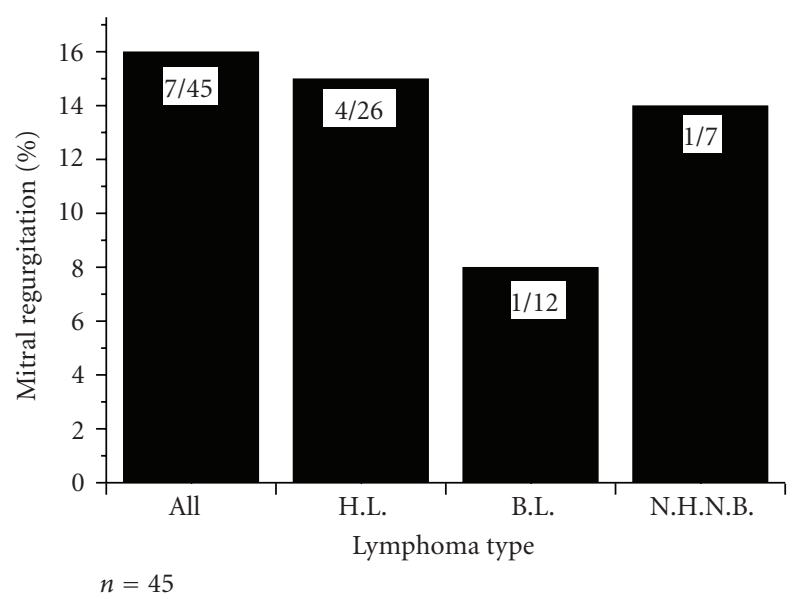

(d)

FIgURE 1: (a) Percentage of survivors with prolonged QTc interval ( $>0.44 \mathrm{~s}$ ) among the lymphoma subgroups. (b) Cardiac index among the lymphoma subgroups. $N=45$, Mean $+\mathrm{SD}$, ANOVA $P<.01$. (c) Left ventricular mass among the lymphoma subgroups. $N=45$, Mean + SD, ANOVA not significant. (d) Percentage of survivors with mitral regurgitation among the lymphoma subgroups. $N=45$. H.D.: Hodgkin's Disease, B.L.: Burkitt's Lymphoma, N.H.N.B.: Non Hodgkin Non Burkitt lymphoma.

cyclophosphamide, dactinomycin, mitomycin, vincristine, bleomycin and methotrexate $[13,18,19]$. Demographic factors include young age, female sex, black race and also trisomy 21 [7]. Among these, our study population was exposed to radiation of up to $4400 \mathrm{cGy}$ (but in most instances less than this) and to polychemotherapy, including vincristine and bleomycin, as dictated by the various treatment protocols. More recently a pathophysiological role for the tyrosine kinase receptor, ErbB2, has been implicated in cardiomyocyte susceptibility to anthracyclines [20].

Again, the total dose of doxorubicin was probably the most important factor in preventing significant cardio toxicity among our population. These findings are consistent with other studies that found that modern protocol therapies using MOPP/ABVD and radiation hold low risk for cardiac toxicity $[21,22]$. 
Leandro et al. [9] suggested that cardiac status following anthracyclines is described by a pattern consistent with a thin-walled, compliant left ventricle with reduced muscle mass performing under above-normal levels of wall stress. Our results are consistent with this description and show left ventricular dimensions at the lower level of normal, with good compliance.

It is surprising that no statistical differences in systolic function and left ventricular mass and thickness were found between the lymphoma type subgroups, in the light of the substantial differences in therapy that these groups received. Although differences in left ventricular mass between BL patients who received anthracyclines and those who did not reach statistical significance, our data may correlate with the aforementioned findings that anthracyclines reduce ventricular mass and reduce left ventricular wall thickness. However, no differences were found between the various lymphoma subgroups, among whom HD patients were exposed both to anthracyclines as well as to radiation. Indeed, these patients exhibited increased cardiac output in comparison to the other lymphoma subgroups. We do not have a clear interpretation for this result. More importantly, cardiac index was at the low range of normal, or slightly below normal, for all groups. Differences between males and females were significant regarding LV mass, a factor that contributes to the greater susceptibility of females to anthracyclines [7], and the greater prevalence of prolonged QTc interval among females.

We found an increased prevalence of mitral valve regurgitation in our study population. An earlier study [23] found mitral valve regurgitation in $24 \%$ of patients. The significance of mitral valve regurgitation in these patients is not entirely clear. Minimal mitral insufficiency, in the absence of valve deformity, is usually of little clinical significance [24]. However, Allen et al. [25] detected an increase in the prevalence of mitral regurgitation among children who had received anthracyclines, $12 \%$ (4 of 34 ) of whom later developed left ventricular systolic dysfunction, concluding that mitral regurgitation might be an early marker of anthracycline-related cardiac dysfunction.

Seventeen percent of the patients we studied demonstrated a prolonged corrected QT interval. Cardiac dysrhythmias are prevalent in survivors of lymphoma treated with anthracyclines [26]. Our findings correlate with those of previous studies, where both QT and QT dispersion were prolonged following treatment with anthracyclines $[27,28]$. A prolonged QT interval may result from myocardial cell damage but does not correlate with decreased contractile function [29, 30]. As dysrhythmias may occur years after termination of treatment and these are potentially lifethreatening, patients should be evaluated at regular followup with a 12-lead ECG $[8,31]$. Although no sudden death events were observed in our population, following our findings of a prolonged Q-T interval in a substantial percentage of the population, it may be suggested that a 24-hour holterECG recording be part of the routine follow-up of these patients.
Coronary artery disease has been reported after radiation therapy to the mediastinum, but not after anthracycline therapy $[1,32]$. This pathology led to significantly increased mortality at 7-year follow-up. Our results did not show resting ECG changes, and there was no premature mortality among our study group on a longer followup period. In the light of findings from other studies, we would suggest periodic ergometry in the long-term follow-up of patients who have undergone radiation therapy [33].

Additional well-described sequelae of radiation therapy to the mediastinum are pericardial effusion and constrictive pericarditis $[34,35]$. We did not note these pathologies to any significant degree in our study population.

The use of possible cardioprotective agents such as dexrazoxane may provide additional protection against longterm cardiac sequelae of anthracyclines, especially in girls $[36,37]$. These agents were not used in our study, and we cannot comment further on their effects.

Limitations. This study has certain limitations. This study was performed as a retrospective analysis of data with inherent limitations. The study group is relatively small and the number of patients in subgroups is small. We did not compare the study group with a control group, as our primary objective was definition of cardiac status among survivors. However, we used well-established norms that are commonly and widely used as the basis for this definition. Further comparisons between the different lymphoma subgroups, provided a comparative basis for the different treatment modalities, and specific comparison between Burkitt lymphoma survivors who had been exposed to anthracyclines and those who had not strengthened this comparison. Echocardiographic parameters such as ejection fraction and fractional shortening are limited by load dependency and constitute basic indicators of global ventricular function. Although other echocardiographic methods are available for assessment of cardiac function, each has its specific limitations and in daily clinical practice many oncologists are more familiar with the traditional parameters used in this study. Likewise assessment of left ventricular mass by echocardiography is limited. This study focused on long-term follow-up with data collected at the last available assessment, and we did not collect data on acute or subacute toxicity.

In summary, in this cohort, relatively few long-term cardiac complications were seen in most patients following low-dose anthracycline therapy used in modern treatment protocols for childhood lymphoma. However, anthracycline cardio toxicity has previously been well established and in the current study, abnormalities were found in mitral valve regurgitation abnormalities, QT prolongation, lownormal cardiac index, and in 2 survivors functional capacity reduction leading to a compromised quality of life. The greater frequency of abnormalities in females requires increased vigilance in this group. These findings necessitate comprehensive and long-term cardiac follow-up of these patients. 


\section{References}

[1] S. L. Hancock, S. S. Donaldson, and R. T. Hoppe, "Cardiac disease following treatment of Hodgkin's disease in children and adolescents," Journal of Clinical Oncology, vol. 11, no. 7, pp. 1208-1215, 1993.

[2] M. A. Grenier and S. E. Lipshultz, "Epidemiology of anthracycline cardiotoxicity in children and adults," Seminars in Oncology, vol. 25, no. 4, pp. 72-85, 1998.

[3] T. J. Porea, Z. E. Dreyer, J. T. Bricker, and D. H. Mahoney, "Evaluation of left ventricular function in asymptomatic children about to undergo anthracycline-based chemotherapy for acute leukemia: an outcome study," Journal of Pediatric Hematology/Oncology, vol. 23, no. 7, pp. 420-423, 2001.

[4] M. T. Meinardi, W. T. A. Van Der Graaf, D. J. Van Veldhuisen, J. A. Gietema, E. G. E. De Vries, and D. TH. Sleijfer, "Detection of anthracycline-induced cardiotoxicity," Cancer Treatment Reviews, vol. 25, no. 4, pp. 237-247, 1999.

[5] R. B. Devereux and N. Reichek, "Echocardiographic determination of left ventricular mass in man. Anatomic validation of the method," Circulation, vol. 55, no. 4, pp. 613-618, 1977.

[6] N. H. Silverman, Pediatric Echocardiography, Williams \& Wilkins, Baltimore, Md, USA, 1st edition, 1993.

[7] J. P. Krischer, S. Epstein, D. D. Cuthbertson, A. M. Goorin, M. L. Epstein, and S. E. Lipshultz, "Clinical cardiotoxicity following anthracycline treatment for childhood cancer: the Pediatric Oncology Group experience," Journal of Clinical Oncology, vol. 15, no. 4, pp. 1544-1552, 1997.

[8] C. Putterman and A. Polliack, "Late cardiovascular and pulmonary complications of therapy in Hodgkin's disease: report of three unusual cases, with a review of relevant literature," Leukemia and Lymphoma, vol. 7, no. 1-2, pp. 109 $115,1992$.

[9] J. Leandro, J. Dyck, D. Poppe et al., "Cardiac dysfunction late after cardiotoxic therapy for childhood cancer," American Journal of Cardiology, vol. 74, no. 11, pp. 1152-1156, 1994.

[10] K. Nysom, K. Holm, S. R. Lipsitz et al., "Relationship between cumulative anthracycline dose and late cardiotoxicity in childhood acute lymphoblastic leukemia," Journal of Clinical Oncology, vol. 16, no. 2, pp. 545-550, 1998.

[11] L. C. M. Kremer, E. C. Van Dalen, M. Offringa, J. Ottenkamp, and P. A. Voûte, "Anthracycline-induced clinical heart failure in a cohort of 607 children: long-term follow-up study," Journal of Clinical Oncology, vol. 19, no. 1, pp. 191-196, 2001.

[12] L. J. Steinherz, P. G. Steinherz, C. T. C. Tan, G. Heller, and M. L. Murphy, "Cardiac toxicity 4 to 20 years after completing anthracycline therapy," Journal of the American Medical Association, vol. 266, no. 12, pp. 1672-1677, 1991.

[13] P. K. Singal and N. Iliskovic, "Doxorubicin-induced cardiomyopathy," New England Journal of Medicine, vol. 339, no. 13, pp. 900-905, 1998.

[14] M. N. Prout, M. J. S. Richards, and K. Ja Chung, "Adriamycin cardiotoxicity in children. Case reports, literature review, and risk factors," Cancer, vol. 39, no. 1, pp. 62-65, 1977.

[15] A. C. Gilladoga, C. Manuel, C. T. Tan, N. Wollner, S. S. Sternberg, and M. L. Murphy, "The cardiotoxcicity of adriamycin and daunomycin in children," Cancer, vol. 37, no. 2, supplement, pp. 1070-1078, 1976.

[16] D. D. Von Hoff, M. W. Layard, and P. Basa, "Risk factors for doxorubicin-induced congestive heart failure," Annals of Internal Medicine, vol. 91, no. 5, pp. 710-717, 1979.

[17] R. A. Minow, R. S. Benjamin, and J. A. Gottlieb, "Adriamycin cardiomyopathy: an overview with determination of risk factors," Cancer Chemotherapy Reports, vol. 6, no. 2, pp. 195201, 1975.

[18] J. R. Kushner, V. L. Hansen, S. P. Hammer, and R. N. Matthews, "Cardiomyopathy after widely seperated courses of adriamycin exacerbated by actinomycin D and mithramycin," Cancer Treatment Reports, vol. 36, pp. 1577-1584, 1975.

[19] P. J. Smith, H. Ekert, K. D. Waters, and R. N. Matthews, "High incidence of cardiomyopathy in children treated with adriamycin and DTIC in combination chemotherapy," Cancer Treatment Reports, vol. 61, no. 9, pp. 1736-1738, 1977.

[20] S. A. Crone, Y. Y. Zhao, L. Fan et al., "ErbB2 is essential in the prevention of dilated cardiomyopathy," Nature Medicine, vol. 8, no. 5, pp. 459-465, 2002.

[21] C. S. LaMonte, S. D. J. Yeh, and D. J. Straus, "Long-term follow-up of cardiac function in patients with Hodgkin's disease treated with mediastinal irradiation and combination chemotherapy including doxorubicin," Cancer Treatment Reports, vol. 70, no. 4, pp. 439-444, 1986.

[22] L. S. Constine, R. G. Schwartz, D. E. Savage, V. King, and A. Muhs, "Cardiac function, perfusion, and morbidity in irradiated long-term survivors of Hodgkin's disease," International Journal of Radiation Oncology Biology Physics, vol. 39, no. 4, pp. 897-906, 1997.

[23] M. B. Lund, H. Ihlen, B. M.R. Voss et al., "Increased risk of heart valve regurgitation after mediastinal radiation for Hodgkin's disease: an echocardiographic study," Heart, vol. 75, no. 6, pp. 591-595, 1996.

[24] J. D. R. Thomson, J. Allen, and J. L. Gibbs, "Left sided valvar regurgitation in normal children and adolescents," Heart, vol. 83, no. 2, pp. 185-187, 2000.

[25] J. Allen, J. D. R. Thomson, I. J. Lewis, and J. L. Gibbs, "Mitral regurgitation after anthracycline treatment for childhood malignancy," Heart, vol. 85, no. 4, pp. 430-432, 2001.

[26] L. J. Steinherz, P. G. Steinherz, and C. Tan, "Cardiac failure and dysrhythmias 6-19 years after anthracycline therapy: a series of 15 patients," Medical and Pediatric Oncology, vol. 24, no. 6, pp. 352-361, 1995.

[27] T. Nousiainen, E. Vanninen, A. Rantala, E. Jantunen, and J. Hartikainen, "QT dispersion and late potentials during doxorubicin therapy for non-hodgkin's lymphoma," Journal of Internal Medicine, vol. 245, no. 4, pp. 359-364, 1999.

[28] C. L. Schwartz, W. L. Hobbie, S. Truesdell, L. C. Constine, and E. B. Clark, "Corrected QT interval prolongation in anthracycline-treated survivors of childhood cancer," Journal of Clinical Oncology, vol. 11, no. 10, pp. 1906-1910, 1993.

[29] J. Leandro, J. Dyck, D. Poppe et al., "Cardiac dysfunction late after cardiotoxic therapy for childhood cancer," American Journal of Cardiology, vol. 74, no. 11, pp. 1152-1156, 1994.

[30] D. L. Keefe, "Anthracycline-induced cardiomyopathy," Seminars in Oncology, vol. 28, no. 4, pp. 2-7, 2001.

[31] R. L. Larsen, R. I. Jakacki, V. L. Vetter, A. T. Meadows, J. H. Silber, and G. Barber, "Electrocardiographic changes and arrhythmias after cancer therapy in children and young adults," American Journal of Cardiology, vol. 70, no. 1, pp. 7377, 1992

[32] J. F. Boivin, G. B. Hutchison, J. H. Lubin, and P. Mauch, "Coronary artery disease mortality in patients treated for Hodgkin's disease," Cancer, vol. 69, no. 5, pp. 1241-1247, 1992.

[33] K. M. Weesner, M. Bledsoe, A. Chauvenet, and M. Wofford, "Exercise echocardiography in the detection of anthracycline cardiotoxicity," Cancer, vol. 68, no. 2, pp. 435-438, 1991. 
[34] R. G. Martin, J. C. Ruckdeschel, and P. Chang, "Radiation related pericarditis," American Journal of Cardiology, vol. 35, no. 2, pp. 216-220, 1975.

[35] D. I. Scott and R. D. Thomas, "Late onset constrictive pericarditis after thoracic radiotherapy," British Medical Journal, vol. 1, no. 6109, pp. 341-342, 1978.

[36] S. E. Lipshultz, R. E. Scully, S. R. Lipsitz et al., "Assessment of dexrazoxane as a cardioprotectant in doxorubicin-treated children with high-risk acute lymphoblastic leukaemia: longterm follow-up of a prospective, randomised, multicentre trial," The Lancet Oncology, vol. 11, no. 10, pp. 950-961, 2010.

[37] G. Chvetzoff, B. Bonnotte, and B. Chauffert, "Anticancer chemotherapy: prevention of toxicityChimiotherapie anticancereuse: prevention de la toxicite," Presse Medicale, vol. 27, no. 39, pp. 2106-2112, 1998. 


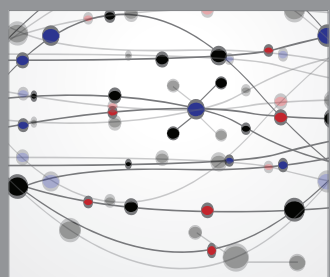

The Scientific World Journal
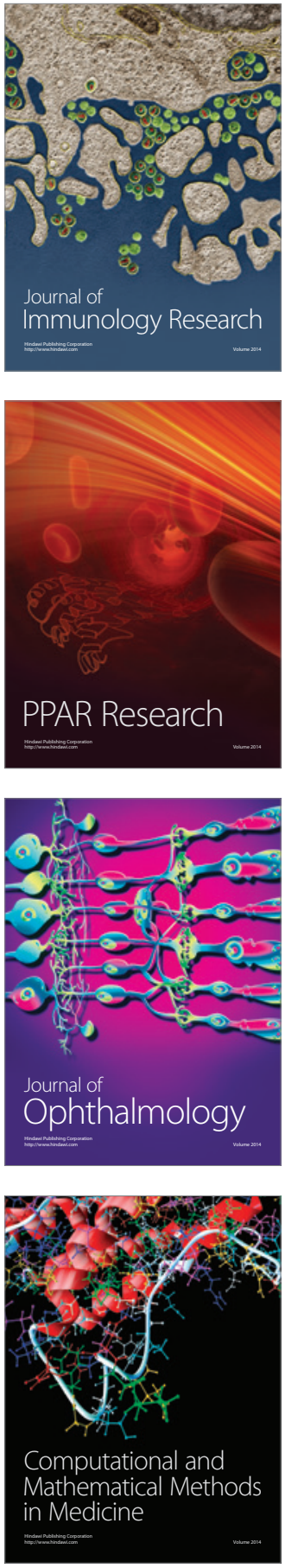

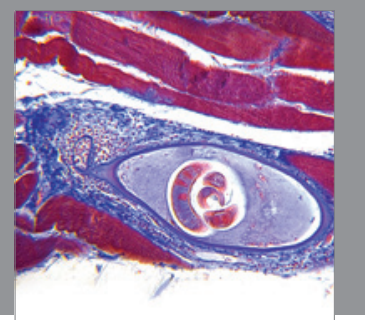

Gastroenterology

Research and Practice
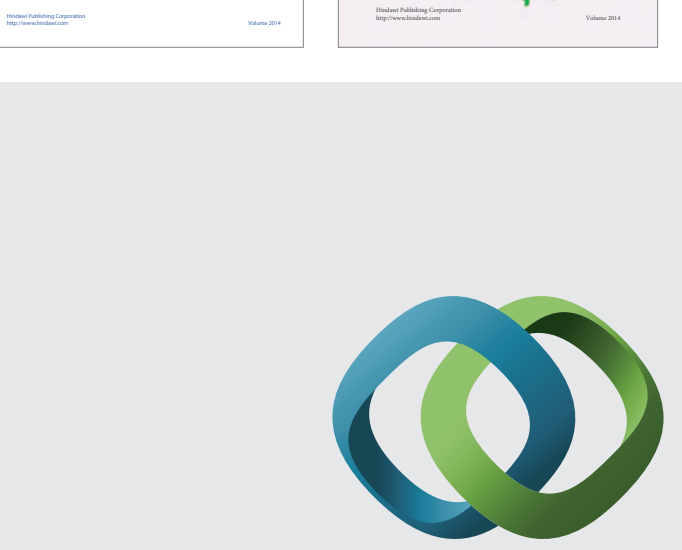

\section{Hindawi}

Submit your manuscripts at

http://www.hindawi.com
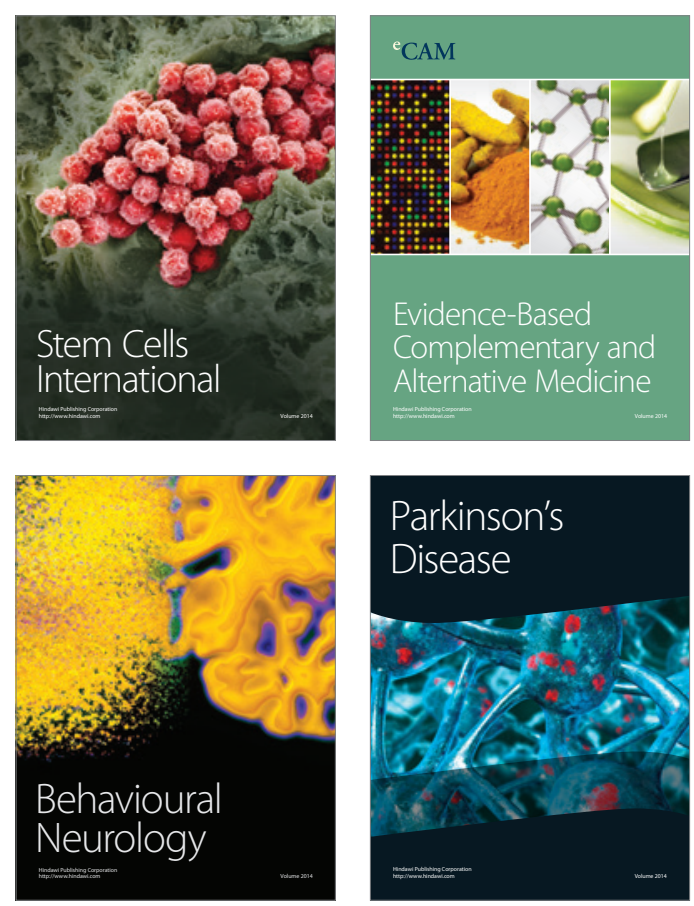

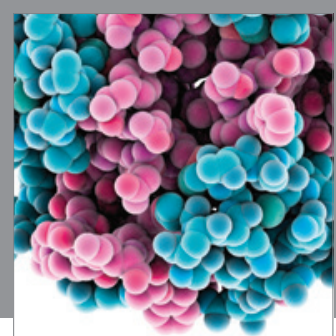

Journal of
Diabetes Research

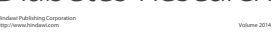

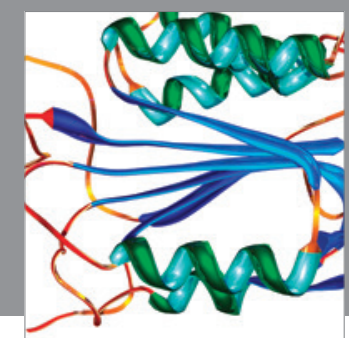

Disease Markers
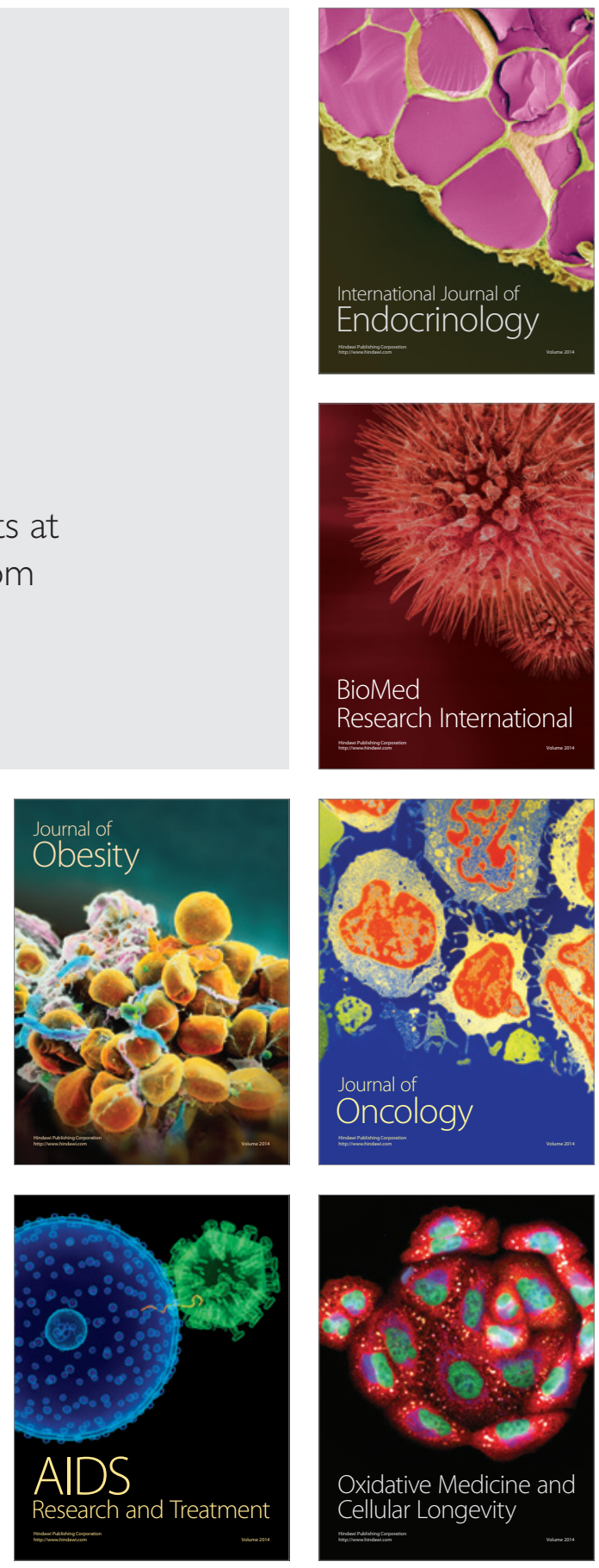